\title{
The extent and costs of reproductive interference among four species of true bug
}

\author{
David M. Shuker · Niall Currie • Tara Hoole • \\ Emily R. Burdfield-Steel
}

Received: 19 March 2014/Accepted: 21 December 2014/Published online: 21 January 2015

(C) The Author(s) 2015. This article is published with open access at Springerlink.com

\begin{abstract}
Reproductive interference arises when individuals of one species engage in reproductive activities with individuals of another, leading to fitness costs in one or both species. Reproductive interference (RI) therefore has two components. First, there must be mis-directed mating interactions. Second, there must be costs associated with these mis-directed interactions. Here we consider RI between four species of true bug in the family Lygaeidae, focusing in particular on the fitness consequences to Lygaeus equestris. The species we consider vary in their relationships with each other, including species in the same or different genus, and with or without natural overlap in their geographic ranges. First we show that inter-specific mating interactions, although not a certain outcome, are common enough to perhaps influence mating behaviour in these species (arising in up to $10 \%$ of inter-specific pairings). Second, we show that reproductive interference can seriously reduce female fitness in L. equestris. Importantly, different species impose different costs of RI on $L$. equestris, with interactions with male Spilostethus pandurus inflicting fitness costs of similar magnitude to the costs of mating with con-specifics. On the other hand, mating interactions with male Oncopeltus fasciatus appear to have no effect on female fitness. In a follow-up experiment, when we allowed competition amongst just females of
\end{abstract}

D. M. Shuker · N. Currie · E. R. Burdfield-Steel ( $\square)$

School of Biology, University of St Andrews, Harold Mitchell

Building, St Andrews, Fife KY16 9TH, UK

e-mail: erb28@st-andrews.ac.uk

D. M. Shuker

e-mail: david.shuker@st-andrews.ac.uk

T. Hoole

Institute of Evolutionary Biology, University of Edinburgh,

West Mains Road, Edinburgh EH9 3JT, UK
S. pandurus and L. equestris, the fitness of the latter was not reduced, arguing more strongly for the role of reproductive interference. However, in our final experiments under mass mating conditions with extended ecological interactions (including scope for competition for resources and cannibalism), the costs of RI were less apparent. Our data therefore suggest that the costs of RI will be contextspecific and may act in concert with, or be swamped by, other ecological effects. We suggest that comparative studies of this sort that both mimic naturally occurring reproductive interference events, and also artificially generate new ones, will be necessary if we are to better understand the ecological and evolutionary significance of reproductive interference.

Keywords Mating behaviour $\cdot$ Lygaeidae $\cdot$ Sexual conflict $\cdot$ Sexual selection

\section{Introduction}

Interspecific interactions can take many forms and play a vital role in shaping evolution. Perhaps the most commonly studied, and best understood, interactions are those that involve predation, herbivory and competition for resources (Begon et al. 2006; Crawley 2009). However, when different species are carrying out the potentially complex behaviours associated with reproduction in the same area, seemingly non-adaptive behaviours, such as reproductive interference, can take place. Reproductive interference (RI) arises when reproductive behaviours occur between individuals of different species and these behaviours result in a loss of fitness for one or both species (Gröning and Hochkirch 2008; Burdfield-Steel and Shuker 2011). RI can take many forms, ranging from signal jamming to 
misdirected courtship and copulation with heterospecifics. These latter forms of RI are thought to entail the highest costs (Rhymer and Simberloff 1996). Relative to hybridisation and its role in understanding gene flow, reproductive isolation and hence speciation (e.g., Arnold 1997), heterospecific mating behaviours that do not produce viable offspring have received comparatively little attention. While inter-species sexual behaviours are well-documented (Andrews et al. 1982; Dame and Petren 2006; de Bruyn et al. 2008), the ecological effects of such interactions are still poorly understood (Gröning and Hochkirch 2008). RI has been implicated in both the exclusion of native species by invasive ones (Butler and Stein 1985) and in determining the ecology and habitat use of naturally co-occurring species (Gröning et al. 2007). If the fitness consequences of RI are sufficient then it may lead to ecological or evolutionary changes that mitigate this cost, including reproductive character displacement (Pfennig and Pfennig 2009). However, more empirical studies of the fitness costs of reproductive interference are needed in order to establish if this is a typical outcome (Gröning and Hochkirch 2008).

Mating behaviour is generally costly for both sexes in terms of time, energy, gamete wastage and in some cases nutrients (Daly 1978), and as such forms part of the overall cost of reproduction (Bell and Koufopanou 1986). However, in many cases of reproductive interference, greater fitness loss is expected to be suffered by females than males (Arnqvist and Rowe 2005). This asymmetry is caused by differences in mating strategies between the sexes, primarily due to dimorphism in gamete size. As females have larger gametes and typically invest more per reproductive event than males, Bateman's principle states that male reproductive success should be limited by mating opportunities, whereas resources available for eggs should limit female reproductive success (Bateman 1948; Trivers 1972). Therefore, males may be selected to mate frequently with all potential mates encountered, even if there is a possibility that some mates are unsuitable, rather than being too discriminating and missing mating opportunities (Reyer 2008). Most types of reproductive interference that involve physical interactions are likely to be costly to both males and females. However, an indiscriminate male's mating strategy could, on balance, still be adaptive if the benefit of fertilising enough conspecific females outweighs the cost of heterospecific mistakes (e.g., Parker and Partridge 1998). Conversely, if females gain all the sperm necessary for lifetime reproduction from a single mating (or a small number of matings: Arnqvist and Nilsson 2000), additional matings may be of little or no benefit, thus selecting for females to be more discriminating or averse to mating.

Reproductive interference is therefore closely associated with sexual conflict over mating (Arnqvist and Rowe
2005). Sexual conflict within a species occurs when males and females differ in their evolutionary optima for a given trait (Parker 1979). In terms of sexual conflict over mating, this typically takes the form of males having higher optimal mating rates than females, as outlined above (Arnqvist and Rowe 2005). Reproductive interference (a between-species phenomenon) may therefore arise from within-species sexual conflict, and indeed feedback into the evolution of optimal mating strategies of males and females. For instance, even though females typically invest more than males in a given reproductive event, it is sometimes the case that females still fail to recognise when mates are unsuitable. If males also lack discrimination in mate choice, then the extent and cost of reproductive interference will be mediated by females' ability to detect and reject heterospecific males. However, females may also suffer costs associated with rejection of potential mates (Kokko and Mappes 2013) and in some species female choice is largely removed due to aggressive male tactics such as sexual harassment and forced copulations (e.g., Shuker and Day 2001, 2002; reviewed by Arnqvist and Rowe 2005). Reproductive interference may thus be both a cause and a consequence of sexual conflict within species.

As previously mentioned, RI can have significant behavioural and ecological impact for the species involved. For example, female spadefoot toads (Spea multiplicata) compromise on male song quality to ensure they mate with the correct species in areas where they co-occur with closely-related heterospecifics (Pfennig 2000). As a result, females in these populations are unable to benefit from the increased fertilization success associated with high-quality males. Sexual harassment by heterospecifics can also impose time and energy costs on those targeted as they attempt to escape, as well as reducing foraging opportunities (Rowe et al. 1996) and access to certain key microhabitats (e.g., preferred oviposition sites for females: Tallamy and Schaefer 1997). Of all the various forms of RI, heterospecific matings have been associated with the greatest costs (Verrell 1994). If post mating barriers are complete (as would be expected in mating attempts between species in different genera) then hybridisation will be impossible and the mating will result in no offspring, whilst still incurring costs. These costs may include sperm competition or displacement, and interruption of fertilisation or gamete development resulting in gamete wastage and inviable eggs being laid (Hochkirch et al. 2007). Damage to females may even occur in cases of mismatch in body size or morphology.

While studies of the ecological effects of RI remain rare, those that do exist frequently concern insects (McLain and Shure 1987; McLain and Pratt 1999; Gröning et al. 2007). McLain and Pratt (1999) found that female lygaeid bugs (Neacoryphus bicrucis), housed following mating with 
males of either the same species or Margus obscurator (a bug in the related Coreidae family), had lower fecundity compared to those housed with females of either species. This cost of heterospecific courtship and harassment may be one of the reasons why the two species are rarely found on the same host plant in nature despite having overlapping ranges (McLain and Pratt 1999). In addition, a series of studies carried out on $N$. bicrucis in the field suggested that indiscriminate, and characteristically aggressive, mating behaviour of the males of the species actively excludes other polyphagous insect species from habitats supporting high N. bicrucis densities (McLain and Shure 1987). As promiscuously mating species with short generation times and straightforward husbandry, bugs in the family Lygaeidae are highly suitable for laboratory studies of intraand inter-specific mating behaviour (Feir 1974; BurdfieldSteel and Shuker 2014). Despite extended copulation durations (e.g., Sillén-Tullberg 1981) they show little, if any, pre-copulatory courtship. Instead copulations are initiated by males, who grasp and mount females, and it is unclear to what extent females must cooperate for genitalia engagement to take place (see Shuker et al. 2006). While evidence has been found for pre-copulatory selection in these insects, it appears to be weak (Burdfield-Steel et al. 2013; Dougherty and Shuker 2014). This may be in part due to selection on males to mate as frequently as possible (assuming a mating system of encounter polyandry: Burdfield-Steel and Shuker 2014), and thus be less selective when assessing potential mates. In addition, previous research has shown that in Lygaeus equestris, mating and sexual harassment by male conspecifics can lead to reduced female longevity (Shuker et al. 2006), suggesting that there is sexual conflict over mating frequency. Furthermore, harassment of $L$. equestris females by males of the species Spilostethus pandurus and Lygaeus creticus has been observed in the laboratory (E. Burdfield-Steel, personal observation; see also Matocq 1990 for another example).

To further explore the possible scope of reproductive interference in lygaeid bugs, we used a geographical and taxonomic hierarchy of four species, in a series of five experiments exploring the frequency of RI and its fitness consequences to our focal species $L$. equestris. First, we compared two populations of the same species $L$. equestris, one from Sicily and one from the Dolomites region of northern Italy. Second, we used the con-gener L. creticus which overlaps in its western distribution with $L$. equestris, including in Sicily where our population was collected. Third, we used S. pandurus, a rather cosmopolitan species across Europe, northern Africa and western Asia. Spilostethus pandurus overlaps the distributions of both L. creticus and L. equestris, including in Sicily for the former and in the Dolomites for the latter. Finally, we used the North American species Oncopeltus fasciatus. This well-known laboratory model species does not share any historical geographic overlap with any of the other species we used (although there have been occasional reports of $S$. pandurus in North America, but it is yet to be considered resident). Although our main aim is to compare the frequency and consequences of putative reproductive interference across these species, with a particular focus on L. equestris, this nesting of populations and species gives us a first glimpse of how inter-specific interactions may map to patterns of sympatry and taxonomic divergence. For example, it may be that closely related species are at a higher risk of RI as they have had less time to evolve robust species recognition mechanisms. Given this, we might expect to see more mating attempts within the genera Lygaeus than across the genus boundary (i.e., with $S$. pandurus or $O$. fasciatus). It has also been suggested that species that co-occur in the wild will have more effective species discrimination mechanisms than those that do not share ranges (Gröning and Hochkirch 2008). In this case we would expect to see more mating attempts between $O$. fasaciatus and the other three species, as $O$. fasciatus would never naturally encounter any of these species.

In our first experiment we investigated levels of heterospecific mating behaviour between the species. We predicted that mating rates will be higher in conspecific pairings and lowest across genera. In our second experiment we looked for reductions in the longevity and egg production of $L$. equestris females as a result of harassment by conspecific and heterospecifics males. If heterospecific males do frequently attempt to copulate with $L$. equestris females then we predicted that they would inflict similar costs to conspecific males. We then compared the lifespan and egg production of females housed with heterospecifics males to those housed with heterospecifics females. If the reduction in both these measures was indeed caused by harassment for matings, rather than some other form of competition, then we expected males to inflict greater costs than females. Finally we looked to see if the patterns observed in the previous experiments could be replicated under mass-mating conditions.

\section{Materials and methods}

\section{Study species}

We used four species of bugs in the family Lygaeidae: $L$. equestris (two populations, derived from bugs collected in Sicily and the Dolomites in northern Italy), L. creticus (from Sicily), S. pandurus (from Sicily), and $O$. fasciatus. The latter species is a North American species that does not naturally co-occur with the other three species (Dingle et al. 1977, 1982). We obtained this species from Dr 
Vernon French from the University of Edinburgh who maintained this species in long-term laboratory culture. The other three species were collected by DMS and colleagues between 2004 and 2008, apart from the Sicily population of L. equestris. This population was collected and then maintained in laboratory culture in Sweden before a new laboratory culture was founded at the University of Leeds by Professor Nina Wedell in 1996. The study population is descended from this previously-called "Leeds" population and has been studied previously alongside the Dolomites population (Shuker et al. 2006).

\section{Insect rearing}

All four species were fed on an ad libitum diet of organic sunflower seeds (Goodness Direct, UK). Stock cages (plastic boxes measuring $30 \times 15 \times 15 \mathrm{~cm}$ with fine mesh over part of the lids) were maintained in an incubator at $29{ }^{\circ} \mathrm{C}$ with a $22: 2 \mathrm{~h}$ light: dark cycle in order to prevent the initiation of reproductive diapause (Shuker et al. 2006). Stock cages were kept in continuous culture with overlapping generations present. The sunflower seeds formed the substrate of the stock cages to a depth of about $3 \mathrm{~cm}$. A piece of non-absorbent cotton wool was added to the cages for bugs to cling on to and hide under. Oviposition was either amongst the sunflower seeds or on this piece of cotton wool. Each week two universal tubes $(30 \mathrm{ml})$ were filled with distilled water and bunged with cotton wool, to act as a water source. Two to three stock cages were kept for each experimental species and stock cages were replaced approximately every 6-8 weeks. A sample of around 60 individuals of all age classes was randomly removed from the original cage to initiate a new one. Eggto-adult development under these conditions is broadly similar for the four species, taking approximately 23-28 days (E. Burdfield-Steel, unpublished data). The bugs used in the experiments were taken from the stock cages when they were large 4th-5th instar nymphs and kept together until eclosion into adults in a similar cage. Nymphs were collected from all of the stock cages to ensure a wide genetic sample and to limit effects of interactions with kin. We checked the nymph cages daily for freshly-eclosed adults which were then kept in single sex groups for 7 days prior to their use in the experiments. This was to ensure that all individuals were mature as these species normally do not become reproductively active until 3-5 days after eclosion.

\section{Experiment 1: The extent of reproductive interference}

To determine the extent to which there are mis-placed mating interactions within- and among-genera, we considered five populations of four species (introduced above).
We set up reciprocal mating trials, pairing males and females from each population with individuals of the opposite sex from each population (a total of 25 possible pairings). Each type of cross was replicated between seven and 23 times, giving a total of 297 pairs.

We sexed and separated newly-eclosed adult bugs into petri dishes $(60 \times 15 \mathrm{~mm})$ where we provided them with sunflower seeds and damp cotton wool in an up-turned lid of a small petri dish, with the water and seeds being replenished as necessary. We kept between four and seven individuals in each dish. After 7 days, individuals were then placed in their experimental pairs.

The experimental mating trials were set up by putting a male and female into a fresh petri dish and then observing them across the next $24 \mathrm{~h}$, before giving them wet cotton wool to prevent death because of dehydration. We then continued to observe them across the next $48 \mathrm{~h}$ or until one of the pair died. The pairs were scanned every 15-20 min for at least $6 \mathrm{~h}$ a day and any mating observed was recorded. These observations were performed between 9 am and $5 \mathrm{pm}$. After $72 \mathrm{~h}$ (or the death of one or both bugs) the trials were terminated.

\section{Experiment 2: The cost of reproductive interference I}

The cost of reproductive interference was quantified by measuring lifetime reduction in fecundity and longevity in L. equestris females when interacting with males of the seed bugs $S$. pandurus and $O$. fasciatus.

Newly eclosed adults were separated by sex and placed in $300 \mathrm{cc}$ plastic boxes ( $S$. pandurus and $O$. fasciatus females were discarded at this point), where they were kept for 7 days (newly-eclosed males do not mate immediately). Mature female L. equestris (Dolomites population) were placed in new $300 \mathrm{cc}$ boxes with an arbitrarily chosen conspecific male from the same population and were oncemated (females have greatly restricted oviposition if they remain unmated). Copulations of over $1 \mathrm{~h}$ were treated as a first mating as sperm transfer is likely to have occurred in that time (Sillén-Tullberg 1981; see also Micholitsch et al. 2000 for the closely-related L. simulans). The male was removed and females were then placed into one of the experimental treatments after copulation. The same $300 \mathrm{cc}$ boxes were used for the experiment, with a layer of seeds covering the base and one small plastic, cotton-wool-bunged water tube. These were replaced weekly or as required. Adult males of all three species were kept by species in $100 \times 100 \mathrm{~mm}$ square petri dishes at relatively low density (5-10 males).

The costs incurred by $L$. equestris females from male harassment were investigated by placing $L$. equestris females in one of four treatments: (1) Once mated females kept in a box alone until death ('equestris alone'), (2) 
Once-mated female with one $L$. equestris male until death ('equestris-equestris'), (3) once-mated female with one $S$. pandurus male ('equestris-pandurus'), (4) once-mated female with one $O$. fasciatus male ('equestris-fasciatus'). Females in all paired treatments (i.e., all except treatment 1) were given 'oviposition holidays' to allow eggs to be oviposited, as high levels of male harassment can prevent females from ovipositing and in some situations can cause highly gravid females to die prematurely due to the buildup of eggs (i.e., becoming "egg-bound"). Each replicate was given two oviposition holidays per week, each consisting of $24 \mathrm{~h}$ in isolation from males. After each oviposition holiday was finished, a male was once again selected arbitrarily from the relevant male stock cages of each species. This ensured that similar costs should be imposed on all replicates, as some males may cause more harassment than others.

Eggs were removed and counted at roughly 3 day intervals, to give a measure of egg production. Female longevity was also recorded, each replicate being scanned daily excluding weekends to check for any dead individuals. A total of 88 females were used in the experiment, with samples sizes ranging from 20 to 25 for each treatment. It was not possible to allow all treatments to continue to run until all females had died, therefore some data were censored, i.e., stopped before death.

\section{Experiment 3: The cost of reproductive interference II}

A second experiment attempted to separate the effect of reproductive interference from foraging competition or other interactions. In this experiment female $L$. equestris were paired with $S$. pandurus females since in the first experiment $S$. pandurus males appeared to have the greatest effect on L. equestris egg production and longevity (see below). This experiment consisted of two treatments (1) two L. equestris females kept together in a $300 \mathrm{cc}$ box as described above; (2) a L. equestris female paired with a $S$. pandurus female. In this experiment egg production was not counted, since there would have been difficulty in differentiating between eggs of the two species. Boxes were replaced weekly to avoid emerging nymphs affecting results. Replicates were scanned as described in "Experiment 1 ". In treatment 1 , date of death was based on the death of the first individual since the two could not be differentiated once the treatment had started.

Experiments $4 \mathrm{a}$ and $4 \mathrm{~b}$ : The cost of reproductive interference in small populations

The focal species for these experiments was $L$. equestris (Dolomites population). Small cages were set up with sunflower seeds and a source of water as before. Each cage contained ten reproductively mature $L$. equestris females which had been housed with a single male for 3 days prior to the start of the experiment to ensure mating. In addition ten "companion" bugs were added to each cage bringing the total number of adult bugs to 20 . There were eight possible treatments; four consisted of males of each species (L. equestris, L. creticus, S. pandurus and O. fasciatus) and four consisted of females from each species. Thus for each species used in the experiment there were two possible treatments, ten males or ten females. Each treatment was replicated five times bringing the total number of cages to 40. The additional females added to the cages were kept in single sex populations after eclosion to ensure they did not lay fertile eggs.

In "Experiment $4 \mathrm{a}$ " the cages were maintained for 7 days in a fixed environmental regime of 22:2 h light:dark cycle and a temperature of $29{ }^{\circ} \mathrm{C}$. During this time water tubes were replaced as needed and any dead bugs removed and their sex and species recorded. At the end of the 7 days, all adults were removed and the cages maintained for a further 10 days to allow any eggs laid to hatch (eggs typically hatch within 7 days of being laid). At the end of this period all cages were frozen to kill the occupants and total number of nymphs at each instar in each cage was counted. The total number of nymphs present in the cage was taken as a measure of the population "fitness". The number of focal L. equestris females found to have died during the course of the experiment was recorded. In the case of the L. equestris female treatment it was not possible to tell if dead females were from the "focal group" or the treatment group (i.e., the companion females) so the number of all L. equestris females that died was recorded and then divided by two as it was assumed that focal and companion females contributed equally to the deaths recorded in this treatment.

We ran this experiment twice (4a and $4 b$ ). The same methods were repeated in "Experiment $4 \mathrm{~b}$ " with one notable difference. During the 7 day experimental period all adult bugs were moved to a new cage every day. All seven cages were then maintained for 10 days as described above. This was done to minimise the opportunity for the adult bugs to cannibalise the eggs (see discussion for further details on egg predation in this experiment). In the first run, the total number of nymphs was recorded for 47 cages with $n=4$ or 5 for each treatment. One cage was discarded due to the presence of $S$. pandurus nymphs and two cages produced no nymphs, which was attributed to a failure of the focal females to mate prior to the start of the experiment. In the second run of this experiment, the total number of nymphs was recorded for 50 cages with $n=5$ for each treatment. 
Experimental analysis

All analyses were carried out using SPSS version 17. Specific details for each experiment are given below.

\section{Experiment 2}

All 'equestris alone' females and all experimental females were used to analyse correlations between abdominal width, dry weight, egg production and longevity. Longevity was analysed using Kaplan-Meier survival analysis. Egg production data were analysed using One-way ANOVA and $t$ tests. ANCOVA was used to incorporate other terms into the model. The whole analysis was repeated with censored data removed (i.e., replicates that were stopped before the focal female died). Although some of the non-normally distributed data were log-transformed, the data in the figures are untransformed for simplicity of interpretation.

\section{Experiment 3}

The difference in longevity between treatments was first analysed with Kaplan-Meier survival analysis. As we did not mark individuals, our experimental design means that any differences in mortality detected between the equestris-equestris and equestris-pandurus treatments could be partly explained by sampling the same L. equestris longevity distribution twice and taking the earliest mortality (for the equestris-equestris treatment) as opposed to only sampling the distribution once (for the equestris-pandurus treatment). This could lead to a biased estimate of true mortality for the first treatment. To get around this, we used the mean and standard deviation of L. equestris longevity in treatment 2 (mean 25.68 days, $\mathrm{SD}=12.91$; this is a conservative estimate as not all females died) to generate randomly two normal distributions with this mean and standard deviation (1000 samples per distribution). We then randomly paired samples from each of the distributions, recording the smaller value. This generated a third normal distribution, representing the sampling bias of our experiment generated by always taking the first individual to die as our estimate of longevity in treatment 1 . The mean of this "bias" distribution was 18.14. We then tested whether our estimate of longevity from the equestrisequestris was significantly different to 18.14 .

\section{Experiments $4 a$ and $4 b$}

A two-way ANOVA was carried out to determine if companion sex, companion species or the interaction between the two had an effect on the fitness of $L$. equestris females. The effect of these treatments on mortality of focal $L$. equestris females was modelled using a GLM with a Poisson distribution with companion sex, companion species and the interaction between the two as the effects in the model.

\section{Results}

\section{Experiment 1}

Reproductive interference occurs uncommonly but repeatedly among heterospecific pairs of our four lygaeid species (Table 1). Across all heterospecific pairings, inter-specific genital coupling was observed in $5.8 \%$ of replicates $(12$ out of 208), varying from 0 to $20 \%$ for specific parings. Generally, where such coupling occurred in a particular cross, it occurred in around $10 \%$ of pairings. Whilst there appears to variation in mating rates across the species, these observations confirm the relative ease with which heterospecific mating attempts occur in these species, at least in the laboratory environment.

\section{Experiment 2}

We turn next to the consequences of these heterospecific interactions. Female L. equestris suffered reduced longevity and fecundity as a result of sexual interactions with conspecific males and hetero-specifics, in particular with male S. pandurus.

In terms of longevity, female lifespan varied significantly between treatments, when censored data were either included or excluded (Log Rank tests: $\chi^{2}=39.18, P \leq 0.001$, and $\chi^{2}=54.53, P \leq 0.001$ respectively; Fig. 1). Female L. equestris kept alone lived longer than both the equestrisequestris females (with censored: $\chi^{2}=14.01, P \leq 0.001$, without censored: $\chi^{2}=12.22, P<0.001$ ) and the equestrispandurus females (with censored: $\chi^{2}=8.45, P \leq 0.001$, without censored: $\left.\chi^{2}=21.96, P<0.001\right)$. Indeed, females in the latter treatment suffered the greatest reduction in longevity. The equestris-fasciatus treatment females did not suffer the same longevity when compared to control females (with censored: $\chi^{2}=0.23, P=0.88$, without censored: $\left.\chi^{2}=0.61, P=0.43\right)$. The difference between the equestrisequestris treatment and equestris-pandurus treatment was non-significant, although approaching significance with censored data included $\left(\chi^{2}=2.84, P=0.09\right)$ and was significant with censored data removed $\left(\chi^{2}=10.40\right.$, $P=0.001$ ).

In terms of fecundity, there was a significant difference in egg production between treatments (Log-transformed egg production, One-way ANOVA: $F_{3,79}=7.045$, $P<0.001$, without censored data: $F_{3,59}=10.22$, $P \leq 0.001$ ), with equestris-only and equestris-fasciatus 
Table 1 The extent of intra- and inter-specific matings across four species of seed bug (two populations of Lygaeus equestris, and single populations of Lygaeus creticus, Spilostethus pandurus and Oncopeltus fasciatus)

\begin{tabular}{|c|c|c|c|c|c|}
\hline & \multicolumn{5}{|l|}{ Female } \\
\hline & L. equestris (Sicily) & L. equestris (Dolomites) & L. creticus & S. pandurus & O. fasciatus \\
\hline \multicolumn{6}{|l|}{ Male } \\
\hline L. equestris (Sicily) & $60 \%(n=10)^{\mathrm{b}}$ & $70 \%(n=10)^{\mathrm{b}}$ & $10 \%(n=10)^{\mathrm{d}}$ & $0 \%(n=10)^{\mathrm{d}}$ & $0 \%(n=10)^{\mathrm{d}}$ \\
\hline L. equestris (Dolomites) & $70 \%(n=10)^{\mathrm{b}}$ & $42 \%(n=12)^{\mathrm{c}}$ & $20 \%(n=10)^{\mathrm{d}}$ & $0 \%(n=10)^{\mathrm{d}}$ & $0 \%(n=10)^{\mathrm{d}}$ \\
\hline L. creticus & $0 \%(n=11)^{\mathrm{d}}$ & $15 \%(n=11)^{\mathrm{d}}$ & $21 \%(n=14)^{\mathrm{d}}$ & $8 \%(n=13)^{\mathrm{d}}$ & $0 \%(n=7)^{\mathrm{d}}$ \\
\hline S. pandurus & $10 \%(n=10)^{\mathrm{d}}$ & $0 \%(n=15)^{\mathrm{d}}$ & $0 \%(n=10)^{\mathrm{d}}$ & $94 \%(n=18)^{\mathrm{a}}$ & $9 \%(n=23)^{\mathrm{d}}$ \\
\hline O. fasciatus & $10 \%(n=10)^{\mathrm{d}}$ & $7 \%(n=15)^{\mathrm{d}}$ & $8 \%(n=13)^{\mathrm{d}}$ & $0 \%(n=10)^{\mathrm{d}}$ & $100 \%(n=15)^{\mathrm{a}}$ \\
\hline
\end{tabular}

The percentage of trials in which the distinctive end-to-end copulation position was observed at least once is given for each reciprocal pairing

a $90-100 \%$

b $50-89 \%$

c $40-49 \%$

d $<40 \%$

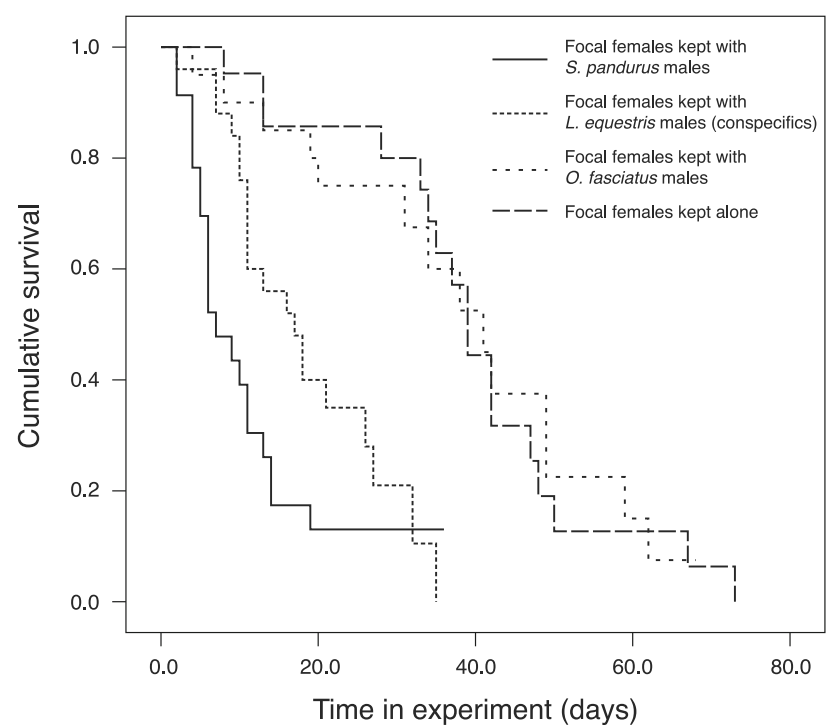

Fig. 1 Interactions with conspecific males or male Spilostethus pandurus reduce the longevity of female Lygaeus equestris. Data are presented as Kaplan-Meier survival curves against time (days), including censored individuals. Solid line focal females kept with $S$. pandurus males, dotted line focal females kept with L. equestris males (conspecifics), dashed line focal females kept with $O$. fasciatus males, extended dashed line focal females kept alone

treatment females producing more females than equestrisequestris or equestris-pandurus females (Fig. 2), and an interaction between egg production and days in treatment (with censored data $F_{3,79}=22.86, P<0.001$, without censored: $F_{3,59}=10.22, P<0.001$ ).

For completeness, longevity and egg production were linked in this experiment. Total eggs produced was correlated with days in treatment for both the equestris alone females (Spearman's rank correlation coefficient: $\left.r_{\mathrm{s}}=0.56, P=0.005\right)$ and across all the females in the experiment $\left(r_{\mathrm{s}}=0.78, P<0.001\right)$.

\section{Experiment 3}

Female $L$. equestris when kept with a con-specific female suffered reduced longevity compared to a female kept with a hetero-specific $S$. pandurus female (Fig. 3). There was a significant difference in days survived between the two treatments, whether including censored data: (Log Rank Test: $\left.\chi^{2}=19.64, P=<0.001\right)$, or with censored data excluded $\left(\chi^{2}=4.93, P=0.026\right)$.

Since bias may have arisen in the estimate of longevity in the equestris-equestris female treatment (see Methods), the longevity estimate from this treatment was compared to a biased estimate of the equestris-pandurus treatment to test whether the two estimates were significantly different. As above, these two estimates are significantly different (one-sample $t$ test: $t_{21}=3.53, P=0.002$ ). Therefore the difference in longevity between treatments was significant even after taking the bias (of one focal female versus two) into account. Thus, the deleterious fitness consequences to female L. equestris of being housed with S. pandurus only occur when the companion bug is male.

\section{Experiment $4 \mathrm{a}$}

Under mass-mating conditions, there was no apparent effect of reproductive interference (or intra-specific costs of mating) in terms of the nymph production of focal female L. equestris. The average number of nymphs produced by $L$. equestris females in this experiment was $276 \pm 136$ (mean \pm S.E.). The results of the two-way ANOVA showed that neither sex (ANOVA: $F_{1,35}=2.69$, $P=0.11)$, species $\left(F_{3,33}=0.50, P=0.68\right)$, nor the interaction between the two $\left(F_{3,33}=0.76, P=0.53\right)$ had any significant effect on the number of nymphs produced by the focal $L$. equestris females. 
Fig. 2 Interactions with conspecific males or male Spilostethus pandurus reduce the fecundity of female Lygaeus equestris. Data are presented as mean $\pm \mathrm{SE}$

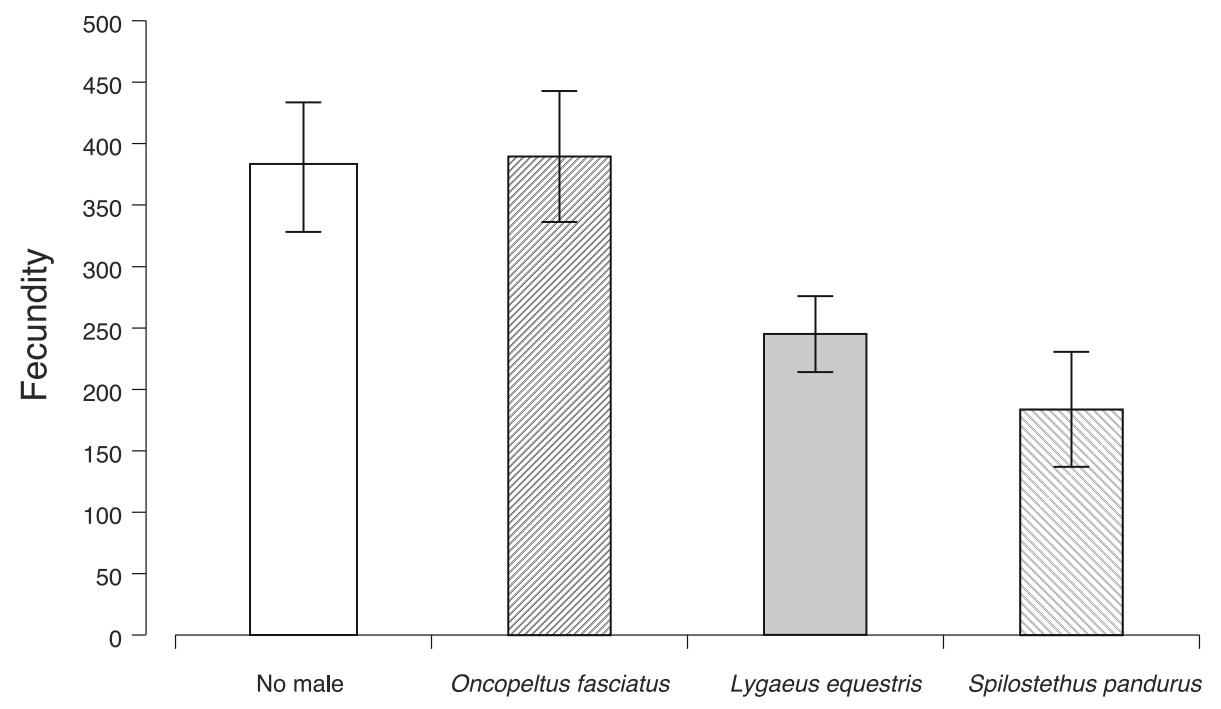

Species identity of male housed with

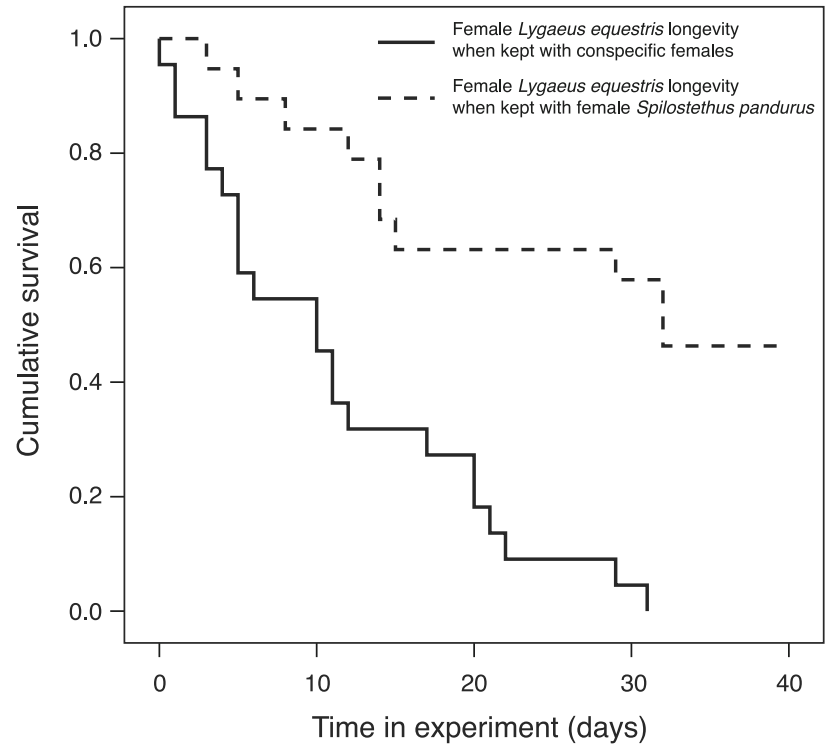

Fig. 3 Female Lygaeus equestris longevity when kept with conspecific females (solid line) or female Spilostethus pandurus (dotted line). Data are presented as Kaplan-Meier survival curves including censored individuals

However, focal females kept with males had reduced longevity compared to focal females kept with other females, regardless of the species identity of the companion bugs. A total of $60 \mathrm{~L}$. equestris females died during the course of the experiment. The average number of focal $L$. equestris females that died when housed with male companion bugs was 2.2 whilst the average number that died when housed with female companion bugs was 1.2 , a difference that is significant (Poisson GLM: LR $=7.51$, $d f=1, P=0.006)$. There was no effect of the species of companion bug $(\mathrm{LR}=4.47, d f=3, P=0.22)$ and no interaction between sex and species of companion bug $(\mathrm{LR}=5.27, d f=3, P=0.15)$. These data do suggest a reduction in longevity when kept with both con- and hetero-specific males, albeit not one that leads to reduced nymph production.

\section{Experiment $4 b$}

In the second run of the experiment, nymph production was influenced by an interaction between sex of the companion bugs and their species identity (ANOVA: $F_{3,36}=5.04$, $P=0.006)$. The main effects of sex and species were not themselves significant $\left(F_{1,39}=0.26, \quad P=0.62\right.$ and $F_{3,36}=1.22, P=0.32$, respectively). This is the result of opposing sex effects in the $O$. fasciatus and $L$. equestris treatments. Lygaeus equestris females had lower nymph production in the presence of females of their own species compared with males; however the pattern was reversed when the treatment bugs were $O$. fasciatus. Fewer nymphs were produced in the presence of $O$. fasciatus males than females (Fig. 4).

In this run, there were however no differences between the number of focal females that died in relation to the sex or species identify of the bugs $(\mathrm{LR}=0.62, d f=1$, $P=0.43$ and $\mathrm{LR}=3.92, d f=3, P=0.27$, respectively) and no significant interaction term $(\mathrm{LR}=4.39, d f=3$, $P=0.22$ ).

\section{Discussion}

Our set of experiments exploring the potential for and possible costs of reproductive interference across four 


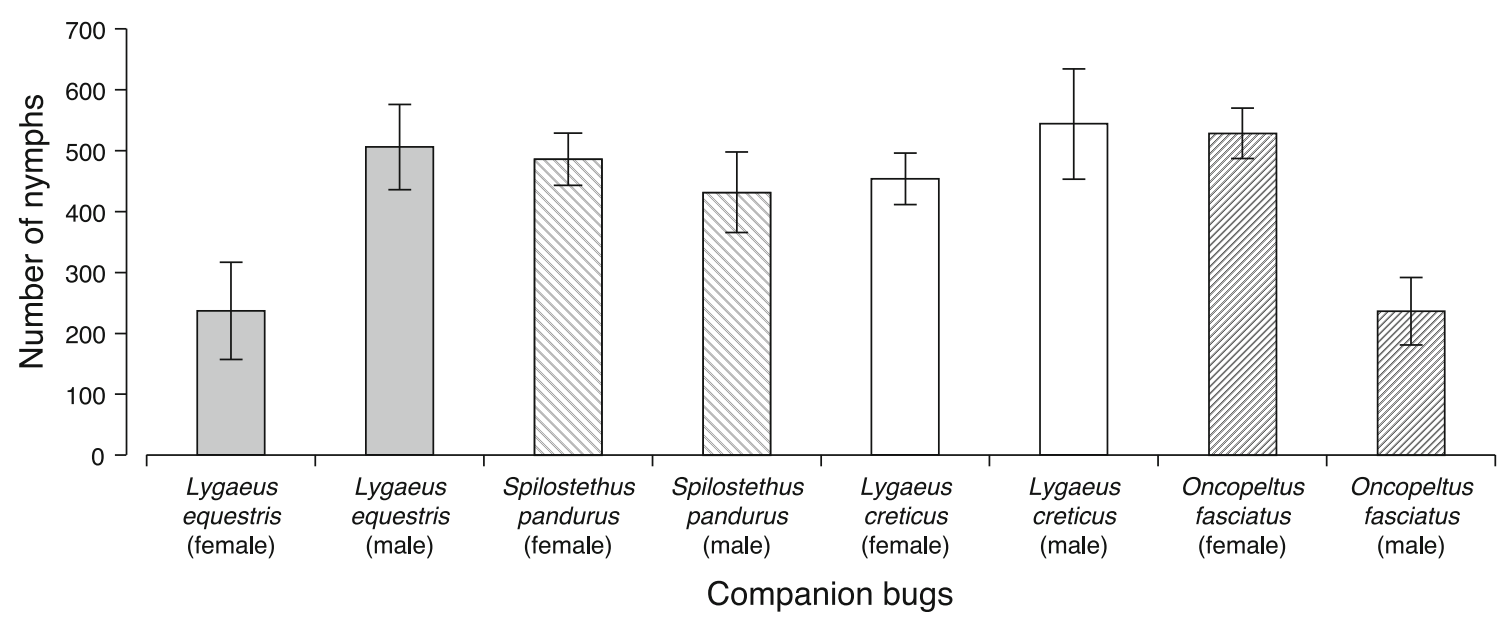

Fig. 4 The mean number of Lygaeus equestris nymphs produced by 10 mated females in the presence of 10 companion bugs from "Experiment $4 \mathrm{~b}$ " (for further details see main text). Data are presented as mean $\pm \mathrm{SE}$

species of seed bug have confirmed both the presence of hetero-specific copulations in this family of bugs (withinand across-genera, for species that do and do not share historic geographical ranges) and that these interactions may be costly to females of our focal species, L. equestris. However, a number of important points have emerged from our experiments. First, hetero-specific mating interactions may be as costly as within-species sexual conflicts over mating ("Experiment 2"; see also Shuker et al. 2006). This result means that viewing reproductive interference as a logical extension of intra-specific sexual conflict over mating (e.g., Arnqvist and Rowe 2005) has some merit. For instance, models of sexual conflict that address reproductive isolation could perhaps be extended to consider reproductive interference more fully (Ribeiro and Spielman 1986; Parker and Partridge 1998). Moreover, the role of sexual conflict in the evolution of polyandry may also be extended to include reproductive interference. For example, convenience polyandry is thought to evolve to reduce the costs of harassment by males in species where mating may be cheaper (or at least the "best of a bad job") than repeatedly rejecting courting and coercing males (e.g., Thornhill and Alcock 1983). But if those matings are with heterospecifics with any appreciable frequency, then convenience polyandry may fail to limit costs. We may therefore expect different evolutionary outcomes of a sexual conflict over mating when heterospecifics and reproductive interference are present or absent. Again, more theoretical modelling may be appropriate here.

Second, in our experiments the costs of reproductive interference depend on the species involved (e.g., male $S$. pandurus impose fitness costs on female $L$. equestris, whereas male $O$. fasciatus in general appear not to). It is unclear yet the extent to which such species-specific effects underpin different consequences of RI in the wild. For instance, reproductive interference is strongly linked to the replacement in Scandinavia of the native crayfish Astacus astacus by the North American crayfish Pasifastacus leniusculus (Westman et al. 2002). However, the same invasive species appears to be replacing the native UK crayfish (Austropotamobius pallipes) via competitive exclusion for refugia (Dunn et al. 2009). Perhaps unsurprisingly therefore, we may well need to understand the mating biology of the particular species involved, plus other aspects of niche partitioning and resource competition, before we will be able to draw general conclusions about the occurrence and evolutionary and ecological consequences of RI (see also Kishi 2014). While the finding that $L$. equestris females experienced RI with $S$. pandurus males but not $O$. fasciatus males seems to support the hypothesis that RI is more likely between closely related species (the exact phylogeny of the Lygaeidae remains unclear, however $S$. pandurus was originally classified as part of the genus Lygaeus and is therefore likely to be more closely related to $L$. equestris than $O$. fasciatus), there could be many other factors contributing to this pattern and further investigation is needed before any conclusions can be drawn.

Third, the experimental context is important. "Experiment 2" gave a far clearer picture than the mass-mating "Experiments 4a and 4b". In "Experiment 2", the costs to female L. equestris of reproductive interference by male $S$. pandurus males was clear (and similar to the costs of conspecific sexual harassment: Shuker et al. 2006). Likewise the absence of such RI when housed with $O$. fasciatus was also clear as females did not suffer any reduction in lifespan or egg production. In "Experiment 4" however there was high variability in the number of nymphs produced within the treatments, which may have contributed to the lack of significant effects detected across treatments. That 
said, the pattern of reduced fitness in the presence of male L. equestris or male $S$. pandurus predicted from the results of "Experiment 2" was not found. Sexual harassment of female $L$. equestris by male $S$. pandurus certainly took place as cross-species matings were observed on several occasions over the course of the experiment. Whether, then, the costs of such interactions were diluted in a larger and more complex environment (for example, were females better able to hide from heterospecifics?), or other interactions, such as resource competition or egg predation, are masking the effects of these costs remains unknown. Egg predation has been previously recorded in some lygaeid species (Sillén-Tullberg 1981; Costa 2006), and $S$. pandurus, particularly females, have been observed preying on L. creticus nymphs in the wild (E. Burdfield-Steel and D. Shuker, personal observation). It is therefore possible that increased egg predation, or even predation of early instar nymphs, by the female companion bugs (who are on average larger than the males of their species) could be imposing fitness costs on the focal females in ways that obscure or remove costs of reproductive interference. Kishi (2014) reviews the links between reproductive interference and cannibalism in classic laboratory studies on inter-specific competition, and our work confirms the insights presented there that the importance of RI will depend on other interspecific interactions, including predation and cannibalism.

In keeping with "Experiment 2", in "Experiment 4a" females in the male L. equestris treatment appeared to have the highest mortality among the focal females, as expected from the finding that $L$. equestris females suffer fitness costs from repeated matings by conspecific males, and that these repeated matings had a greater fitness cost than sexual harassment alone (Shuker et al. 2006), but the differences among male-companion treatments was not significant (data not shown). Instead, being kept with males, regardless of species identity, was costly in terms of increased mortality when compared to being kept with females. It is especially interesting though that this increased mortality does not seem to correlate with reduced "fitness" in this study (an increasingly common finding: Arnqvist and Nilsson 2000; Hunt et al. 2004). One possible explanation might be that the period of time for which the reproductive output of the focal females was measured during this experiment was insufficient to capture the costs that sexual harassment and reproductive interference can impose on a female over the course of her lifespan.

Our final experiment ("Experiment $4 \mathrm{~b}$ ") was designed to limit opportunities for egg predation to some extent, by changing rearing boxes more frequently. The higher average numbers of nymphs produced by the focal females during the course of this experiment indicates this was at least partially successful. While the interaction between sex and species was found to have a significant effect on the number of nymphs produced, the pattern observed did again not correspond with previous findings. The lack of significant trends in mortality may be a result of the reduced number of deaths overall during the course of this experiment compared to "Experiment 2", but overall the results again suggest that experimental details influence the appearance of RI.

In summary, the potential for reproductive interference exists across the species of lygaeid seed bug studied here, reproductive interference that in certain circumstances can impose fitness costs similar to that imposed by con-specific sexual conflict over mating. However, our data also strongly suggest that the effects of RI are likely to be highly ecologically contingent (or in the laboratory, experiment-specific). The challenge for incorporating reproductive interference more broadly into future studies of the ecology and evolution of reproductive behaviour will be to unpick this context-dependence.

Acknowledgments We are very grateful to the editors of this special issue for allowing us to submit this paper as part of that, and also for their patience in waiting for the manuscript. We are also very grateful to the anonymous reviewers for their constructive and generously given advice. ERB-S is supported by a Natural Environment Research Council (NERC) studentship and DMS was in part supported by a NERC Advanced Fellowship.

Open Access This article is distributed under the terms of the Creative Commons Attribution License which permits any use, distribution, and reproduction in any medium, provided the original author(s) and the source are credited.

\section{References}

Andrews RH, Petney TN, Bull CM (1982) Reproductive Interference between 3 parapatric species of Reptile Tick. Oecologia $52: 281-286$

Arnold ML (1997) Natural hybridization and evolution. Oxford University Press, Oxford

Arnqvist G, Nilsson T (2000) The evolution of polyandry: multiple mating and female fitness in insects. Anim Behav 60:145-164

Arnqvist G, Rowe L (2005) Sexual conflict. Princeton University Press, Princeton

Bateman A (1948) Intra-sexual selection in Drosophila. Heredity 2:349-368

Begon M, Townsend CR, Harper JL (2006) Ecology: from individuals to ecosystems. Blackwell Publishing, Oxford

Bell G, Koufopanou V (1986) The cost of reproduction. Oxf Surv Evolut Biol 3:83-131

Burdfield-Steel ER, Shuker DM (2011) Reproductive interference. Curr Biol 21:R450-R451

Burdfield-Steel ER, Shuker DM (2014) The evolutionary ecology of the Lygaeidae. Ecol Evol 4:2278-2301

Burdfield-Steel ER, Dougherty LR, Smith LA, Collins LA, Shuker DM (2013) Variation in social and sexual behaviour in four species of aposematic seed bugs (Hemiptera: Lygaeidae): the role of toxic and non-toxic food. Behav Process 99:52-61 
Butler MJ, Stein RA (1985) An analysis of the mechanisms governing species replacements in crayfish. Oecologia 66:168-177

Costa JT (2006) The other insect societies. Harvard University Press, Harvard

Crawley MJ (2009) Population dynamics of natural enemies and their prey. In: Crawley MJ (ed) Natural enemies. Blackwell Scientific Publications, Oxford, pp 40-89

Daly M (1978) The cost of mating. Am Nat 112:771-774

Dame EA, Petren K (2006) Behavioural mechanisms of invasion and displacement in Pacific island geckos (Hemidactylus). Anim Behav 71:1165-1173

de Bruyn PJN, Tosh CA, Bester MN (2008) Sexual harassment of a king penguin by an Antarctic fur seal. J Ethol 26:295-297

Dingle H, Brown CK, Hegmann JP (1977) Nature of genetic variance influencing photoperiodic diapause in a migrant insect, Oncopeltus fasciatus. Am Nat 111:1047-1059

Dingle H, Blau W, Brown C, Hegmann JP (1982) Population crosses and the genetic structure of milkweed bug life histories. In: Dingle H, Hegmann JP (eds) Evolution and genetics of life histories. Springer-Verlag, New York, pp 209-229

Dougherty L, Shuker D (2014) Pre-copulatory sexual selection in the seed bug Lygaeus equestris: a comparison of choice and nochoice paradigms. Anim Behav 89:207-214

Dunn JC, McClymont HE, Christmas M, Dunn AM (2009) Competition and parasitism in the native White Clawed Crayfish Austropotamobius pallipes and the invasive Signal Crayfish Pacifastacus leniusculus in the UK. Biol Invasions 11:315-324

Feir D (1974) Oncopeltus fasciatus-research animal. Annu Rev Entomol 19:81-96

Gröning J, Hochkirch A (2008) Reproductive interference between animal species. Q Rev Biol 83:257-282

Gröning J, Lucke N, Finger A, Hochkirch A (2007) Reproductive interference in two ground-hopper species: testing hypotheses of coexistence in the field. Oikos 116:1449-1460

Hochkirch A, Gröning J, Bucker A (2007) Sympatry with the devil: reproductive interference could hamper species coexistence. J Anim Ecol 76:633-642

Hunt J, Bussiere LF, Jennions MD, Brooks R (2004) What is genetic quality? Trends Ecol Evol 19:329-333

Kishi S (2014) Reproductive interference in laboratory competition experiments. Popul Ecol. doi:10.1007/s10144-014-0455-0

Kokko H, Mappes J (2013) Multiple mating by females is a natural outcome of a null model of mate encounters. Entomol Exp Appl 146:26-37

Matocq A (1990) Intergeneric mating of Lygaeinae. Entomologiste (Paris) 46:299-300

McLain DK, Pratt AE (1999) The cost of sexual coercion and heterospecific sexual harassment on the fecundity of a hostspecific, seed-eating insect (Neacoryphus bicrucis). Behav Ecol Sociobiol 46:164-170
McLain DK, Shure DJ (1987) Pseudocompetition-interspecific displacement of insect species through misdirected courtship. Oikos 49:291-296

Micholitsch T, Krugel P, Pass G (2000) Insemination and fertilization in the seed bug Lygaeus simulans (Heteroptera : Lygaeidae). Eur J Entomol 97:13-18

Parker GA (1979) Sexual selection and sexual conflict. In: Blum MS, Blum BN (eds) Sexual selection and reproductive competition in insects. Academic Press, New York, pp 23-166

Parker GA, Partridge L (1998) Sexual conflict and speciation. Philos Trans R Soc B Biol Sci 353:261-274

Pfennig KS (2000) Female spadefoot toads compromise on mate quality to ensure conspecific matings. Behav Ecol 11:220-227

Pfennig KS, Pfennig DW (2009) Character displacement: ecological and reproductive responses to a common evolutionary problem. Q Rev Biol 84:253-276

Reyer H-U (2008) Mating with the wrong species can be right. Trends Ecol Evol 23:289-292

Rhymer JM, Simberloff D (1996) Extinction by hybridization and introgression. Annu Rev Ecol Syst 27:83-109

Ribeiro JMC, Spielman A (1986) The satyr effect: a model predicting parapatry and species extinction. Am Nat 128:513-528

Rowe L, Krupa JJ, Sih A (1996) An experimental test of conditiondependent mating behavior and habitat choice by water striders in the wild. Behav Ecol 7:474-479

Shuker DM, Day TH (2001) The repeatability of a sexual conflict over mating. Anim Behav 61:755-762

Shuker DM, Day TH (2002) Mate sampling and the sexual conflict over mating in seaweed flies. Behav Ecol 13:83-86

Shuker DM, Ballantyne GA, Wedell N (2006) Variation in the cost to females of the sexual conflict over mating in the seed bug, Lygaeus equestris. Anim Behav 72:313-321

Sillén-Tullberg B (1981) Prolonged copulation-a male post-copulatory strategy in a promiscuous species, Lygaeus equestris (Heteroptera, Lygaeidae). Behav Ecol Sociobiol 9:283-289

Tallamy DW, Schaefer C (1997) Maternal care in the Hemiptera: ancestry alternatives and current adaptive value. In: Choe JC, Crespi BJ (eds) The evolution of social behaviour in insects and arachnids. Cambridge University Press, Cambridge, pp 94-115

Thornhill R, Alcock J (1983) The evolution of insect mating systems. Harvard University Press, Cambridge

Trivers R (1972) Parental investment and sexual selection. In: Campbell B (ed) Sexual selection and the descent of man. Aldine-Atherton, Chicago, pp 136-179

Verrell PA (1994) Is decreased frequency of mating among conspecifics a cost of sympatry in salamanders? Evolution 48:921-925

Westman K, Savolainen R, Julkunen M (2002) Replacement of the native crayfish Astacus astacus by the introduced species Pacifastacus leniusculus in a small, enclosed Finnish lake: a 30-year study. Ecography 25:53-73 